P143 (continued)

primary aim of this study, to examine the relationship between gardening and gut microbiome composition.

Funding: USDA

\section{P143 Analyzing Costs Associated with Implementation of a Nutrition Program in Early Childhood Education}

Janna Martin, BA,BS, jmartin@uams.edu, University of Arkansas for Medical Sciences, 4301 W Markham St, Slot 530, Little Rock, AR, 72205; Taren Swindle, PhD, MS, University of Arkansas for Medical Sciences

Background: Cost analysis is a tool used to summarize and report on individual elements of costs to implementing an innovation. We used cost analysis to track and compare costs of provision of 2 implementation strategies to support an evidence-based nutrition curriculum in the Head Start (HS) setting.

Objective: To compare how much it cost, per classroom per year, to deliver the basic (ie, training and reminders) and enhanced implementation support (ie, a package of 8 implementation strategies).

Study Design, Setting, Participants: We partnered with a HS program in an urban area of a Southern state with 9 sites and 38 classrooms. We used stratified randomization to assign sites to participate in either the basic or the enhanced implementation of the nutrition curriculum. All sites received the same 6-hour training and implemented the curriculum with either basic or enhanced implementation support.

Measurable Outcome/Analysis: Cost accounting methods allowed for a comparison of time and materials allocated to each group. Our primary outcome of cost included fixed (eg, costs of trainings, materials, printing) and variable (eg, duration of facilitation, number of incentives, travel) costs for both strategies. Facilitators logged activities using a smartphone application.

Results: Estimated costs per classroom, per year were \$35 for basic and \$261 for enhanced implementation support. This reflects average delivery of 4 incentives and 2 resources per classroom, a total of 39.2 in-person and 20.4 virtual facilitation hours to enhanced classrooms. In-person facilitation accounted for $25 \%$ of the enhanced costs; travel $23 \%$; and the tailored incentives and educational materials $21 \%$.

Conclusions: These data, paired with outcome data, will help understand the relative cost in improving implementation outcomes (eg, fidelity, acceptability) between groups, and will inform scalability and sustainability efforts for future uptake of the intervention.

Funding: USDA Lincoln Health; NIH.

\section{P144 A Comprehensive Community Partnership Model for Improving Nutrition Among SNAP- Eligible Families in Early Education Settings}

Razan Sahuri, MPH, rsahuri@umd.edu, University of Maryland Extension, 3300 North Ridge Rd, Suite 240,
Ellicott City, MD, 21043; Erin Duru, MHS, RD, LDN, University of Maryland Extension; Nicole Walker, PhD, University of Maryland Extension

Objective: One in 4 U.S. children are overweight or obese before they reach kindergarten. Early intervention encourages the development of positive behaviors, including healthy eating, that are necessary for young children's healthy growth, development and prevention from chronic diseases. This model highlights an innovative community partnership model to increase the consumption of fruits and vegetables among low-income preschool families.

Use of Theory or Research: Maryland SNAP-Ed implements an innovative partnership model with multilevel interventions for children and their families; as well as policy, systems, and physical environment (PSE) changes which target each level of the socio-ecological model.

Target Audience: Low-income preschool children and their families in Howard County, Maryland.

Program Description: This unique collaboration with the county school system; Head Start; Women, Infants, and Children (WIC) program; Food Bank; Farmers' Markets; and the Local Health Improvement Coalition leverages resources to improve access to and consumption of healthy foods. Children and families receive nutrition education and exposure to programs linking to gardens, the grocery store, and farmers' markets.

Evaluation Methods: Maryland SNAP-Ed's comprehensive evaluation model assesses individual-level impacts through participant surveys and PSE changes through post-program year reports.

Results: Maryland SNAP-Ed programming is associated with improved individual health behaviors, including increased consumption and purchasing of healthy foods. Early education sites in Howard County have also established health-promoting PSEs, such as healthy classroom celebrations, limited availability of sugar-sweetened beverages, and increased variety of healthy foods offered during family events and on-site food pantries, that encourage positive nutrition behaviors.

Conclusions: Maryland SNAP-Ed's holistic approach with multiple county collaborators establishes a sustainable county-wide framework that increases access to fruits and vegetables for preschool families. This poster shares a detailed sustainable approach for others to replicate.

Funding: Supplemental Nutrition Assistance Program Education.

\section{P145 Results of Using Improvised Kitchen Spaces to Teach Skills-Based Nutrition in Schools}

Amelia Huelskamp,PhD, huelskampa@uncw.edu, University of North Carolina Wilmington, 601 S. College Rd, Wilmington, NC 28403 